\title{
Prospect for a compact strontium optical lattice clock
}

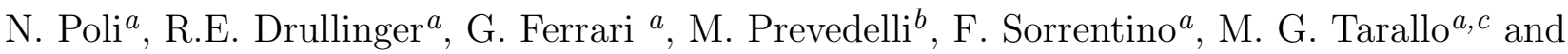 \\ G. M. Tino ${ }^{a}$ \\ ${ }^{a}$ Dipartimento di Fisica and LENS Istituto Nazionale Fisica della Materia - CNR \\ Istituto Nazionale di Fisica Nucleare, Sezione di Firenze \\ Università di Firenze, Polo Scientifico, 50019 Sesto Fiorentino, Italy \\ ${ }^{b}$ Dipartimento di Chimica Fisica, Università di Bologna \\ Via del Risorgimento 4, 40136 Bologna, Italy \\ ${ }^{c}$ Dipartimento di Fisica Applicata - Istituto Nazionale di Fisica Nucleare, Sezione di Pisa \\ Largo Pontecorvo 3, 56100 Pisa (Italy)
}

\begin{abstract}
We report on our progress toward the realization of a compact optical frequency standard referenced to strontium intercombination lines. Our current setup allows the production of ultracold Sr atoms in hundreds of ms. For high resolution spectroscopy of the ${ }^{1} \mathrm{~S}_{0^{-}}{ }^{3} \mathrm{P}_{0}$ doubly forbidden transition we have prepared a $698 \mathrm{~nm}$ clock laser stabilized on a high finesse, symmetrically suspended cavity and a high power 813 nm light source for the optical lattice trap at the magic wavelength. Due to their compactness, reliability, and low power consumption, semiconductor laser sources represent the best choice for the development of compact and transportable devices for application both on Earth and in Space. A new Sr trapping and cooling experimental setup is also presented.
\end{abstract}

Keywords: optical clock, semiconductor laser source

\section{INTRODUCTION}

Optical frequency standards based either on alkali-earth neutral atoms trapped in optical lattices or single trapped ions have demonstrated tremendous advantages in terms of short term stability (approaching $10^{-15}$ at $1 \mathrm{~s}^{1,2}$ ) and ultimate accuracy (approaching $10^{-17}$ level $^{3}$ ). Increasing interest in the realization of compact and transportable optical clocks, for applications on Earth and Space experiment, has recently grown. A number of proposals for

Further author information: (Send correspondence to G. M. Tino)

N. Poli: E-mail: poli@lens.unifi.it, Telephone: +39055 4572503

G. M. Tino: E-mail: Guglielmo.Tino@fi.infn.it, Telephone: +39055 4572034

Time and Frequency Metrology, edited by R. Jason Jones,

Proc. of SPIE Vol. 6673, 66730F, (2007) - 0277-786X/07/\$18 - doi: 10.1117/12.739057 


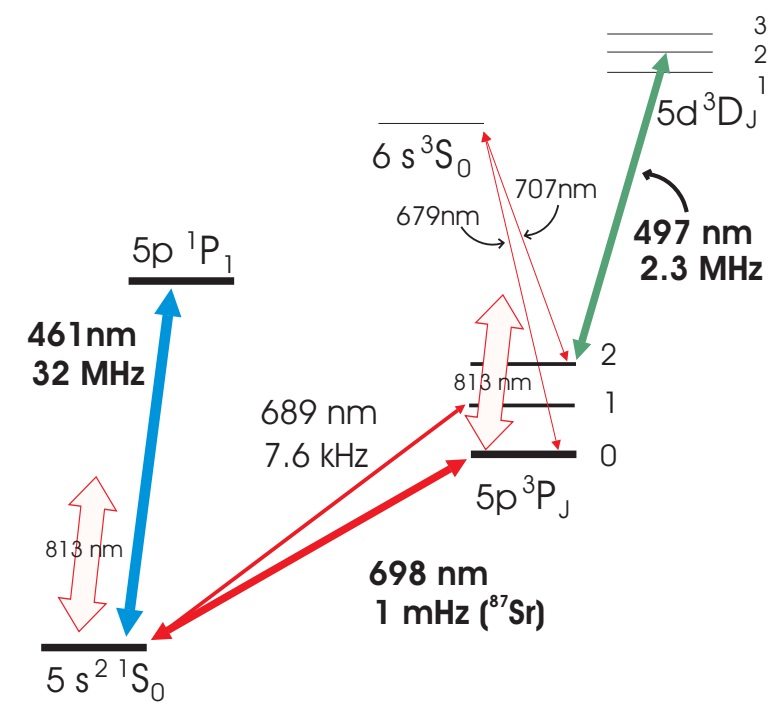

Figure 1. Simplified energy levels and interesting optical transition for strontium optical clock. All the transitions are in the visible or near infrared and can be reached with semiconductor laser sources.

precision tests of General Relativity ${ }^{4-7}$ and for application in Deep Space navigation, ${ }^{8}$ require compact and ultra-stable optical frequency references. While many optical clocks based on either neutrals or single ions have already been realized and tested, these systems are far from compact, generally requiring an entire laboratory room. At least four different wavelengths are necessary both for single ions and optical lattice clocks (sometimes in the UV region of the spectrum) involving frequency doubling or quadrupling stages starting from high power infrared sources. Among all candidates for optical frequency standards, neutral strontium has the advantage that all of the transitions required for clock operation (cooling and trapping, repumping and clock interrogation) are in the visible or near infrared part of the spectrum (see Fig.1). This represents a huge advantage since these wavelengths are easy to produce with compact, reliable and typically inexpensive semiconductor laser sources, which reduces the setup complexity.

Here we present an experimental setup we are developing for realization of an optical frequency standard based on neutral strontium atoms. In partcular a new setup for cooling and trapping strontium is under construction. Additionaly two new compact laser sources have been realized: the first is a clock $698 \mathrm{~nm}$ frequency stabilized laser source, while the second is an infrared $813 \mathrm{~nm}$ high power infrared source to trap atoms in the optical lattice at the magic wavelength. In section 2 we present the experimental apparatus for trapping and cooling strontium, while in sections 3 and 4 new high finesse cavities for stabilization of the clock $698 \mathrm{~nm}$ laser are described. Finally in section 5 we present the infrared $813 \mathrm{~nm}$ laser source used to trap pre-cooled strontium atoms in the dipole trap at the magic wavelength. 


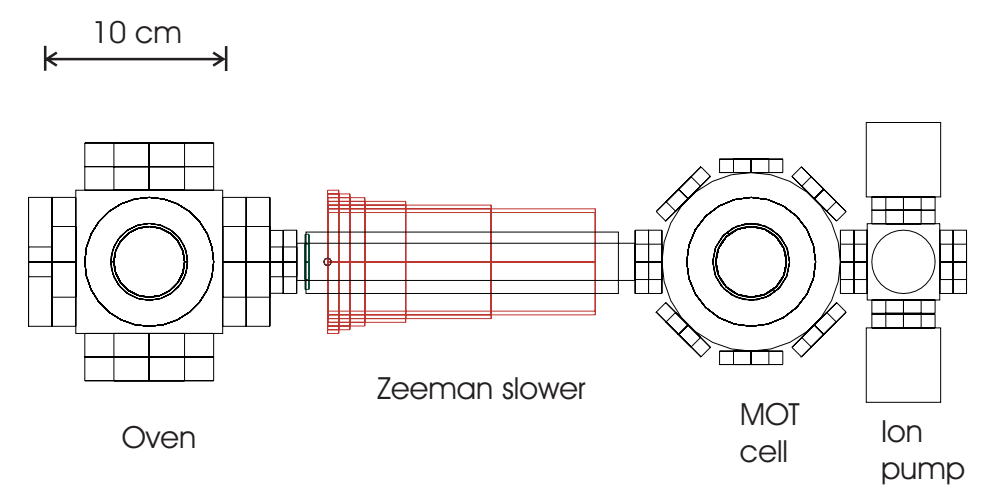

Figure 2. Design for a compact Sr cooling and trapping apparatus. The dimension of the setup is $500 x 150 x 130 \mathrm{~mm}^{3}$.

\section{COOLING AND TRAPPING STRONTIUM}

The basic experimental setup used to trap and cool strontium atoms has previously been described in detail in. ${ }^{9-11}$ In Fig. 2 we show a design of a new compact version of the setup.

Strontium vapors is generated from a Sr dispenser in the oven region. Atoms are then decelerated in a $20 \mathrm{~cm}$ long Zeeman slower, and finally trapped and cooled in the MOT chamber. For this system, two $8 \mathrm{l} / \mathrm{s}$ ion pumps would be sufficient to maintain a background pressure below $10^{-8}$ Torr inside the main chamber. The dimension of the setup is $500 \times 150 \times 130 \mathrm{~mm}^{3}$.

With the current setup it is possible to cool and trap about $10^{78} \mathrm{Sr}$ atoms at $\mu \mathrm{K}$ temperatures in hundreds of ms. The laser sources for trapping and cooling are based on semiconductor laser diodes. For the first cooling and trapping stage on the ${ }^{1} \mathrm{~S}_{0^{-}}{ }^{1} \mathrm{P}_{1}$ transition we used two frequency doubled infrared lasers that deliver repectively $200 \mathrm{~mW}$ at $461 \mathrm{~nm}$ (922 nm extended cavity diode laser amplified with a tapered amplifier and doubled in doubling cavity with PPKTP crystal) and $1 \mathrm{~mW}$ at $497 \mathrm{~nm}$ (994 nm ECDL frequency doubled with $\mathrm{KNbO}_{3}$ crystal). For the second stage cooling on the ${ }^{1} \mathrm{~S}_{0^{-}}{ }^{3} \mathrm{P}_{1}$ transition, a frequency stabilized ECDL at $689 \mathrm{~nm}$ is employed. To detect the clock transition with maximum $\mathrm{S} / \mathrm{N}$ ratio with shelving technique, it is possible to use additional repumpers at $679 \mathrm{~nm}$ and at $707 \mathrm{~nm}$ that also could be realized with simple ECDLs.

An estimation of power consumption, volume and weight for the compact strontium clock (vacuum, electronics and optics) is respectively of the order of $140 \mathrm{dm}^{3}, 90 \mathrm{~kg}$, and $90 \mathrm{~W} \cdot{ }^{12}$

\section{SYMMETRICALLY SUPPORTED HIGH FINESSE CAVITIES}

The high finesse cavity is realized with a $10 \mathrm{~cm}$ long ULE (High grade Corning 7972 glass) spacer with two optically contacted $\mathrm{SiO}_{2}$ mirrors. 
The geometry of the spacer has been optimized with the help of finite element analysis (FEM) to reduce the effect of the deformation induced by vibrations coming from the optical table. ${ }^{13,14}$ The spacer has a "mushroom slotted shape" obtained by cutting an ULE cylinder along its longitudinal z-axis. The positions of the cut along horizontal and vertical directions are respectively $x=46.19 \mathrm{~mm}$ and $y=-6.08 \mathrm{~mm}$.

The cavity is supported horizontally under vacuum $\left(10^{-8}\right.$ Torr, maintained with a $20 \mathrm{l} / \mathrm{s}$ ion pump) with two aluminium arms connected by three low-expansion iron shafts. The effective supporting points are four square areas (about $2 \mathrm{~mm}^{2}$ in size) with Viton square pieces $(0.5 \mathrm{~mm}$ thickness) between the aluminium supporting points and the ULE spacer surface. The vacuum chamber has been built with thick aluminium walls $(5 \mathrm{~cm})$ to increase the thermal inertia of the system. The temperature of the outside surface of the vacuum can is actively stabilized at $25{ }^{\circ} \mathrm{C}$ by controlling the current passing through a high resistance (Alumel) cable wound around the can itself. The measured time constant of the system in that condition is about 3 hours, while the residual temperature error is below $50 \mathrm{mK}$ peak to peak. Two identical cavities have been mounted inside independent vacuum systems to allow performance tests of the frequency stabilized $698 \mathrm{~nm}$ laser.

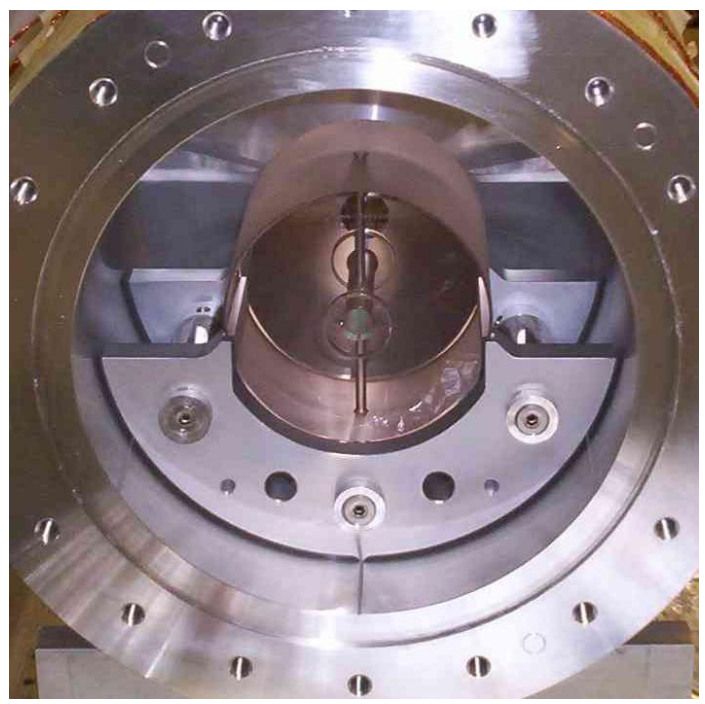

Figure 3. Picture of the cavity inside the thick aluminum vacuum chamber (30 $\mathrm{cm}$ ext. diam.). The cavity sits on four $2 \mathrm{~mm}^{2}$ areas on the aluminum support plate. To equalize the forces, small pieces of Viton have been interposed between the cavity and the aluminum supporting areas. The aluminum plates, connected with three iron shafts, lie on the internal surface of the vacuum can on three points along three grooved lines $(0.5 \mathrm{~mm}$ depth) that define the vertical direction inside the vacuum can.

In Fig. 3 we show a picture of the cavity sitting on the aluminum-iron support, inside the cylindrical vacuum chamber. With the help of finite element analysis (FEM) simulation we checked the cavity static distortion induced by accelerations in both vertical and horizontal directions, as a function of the position of the supporting points along the longitudinal z-axis of the cavity. In Fig.4 we present the calculated frequency displacements due to a relative displacement of the center of the two mirrors, and the residual mirror tilt per unitary vertical 

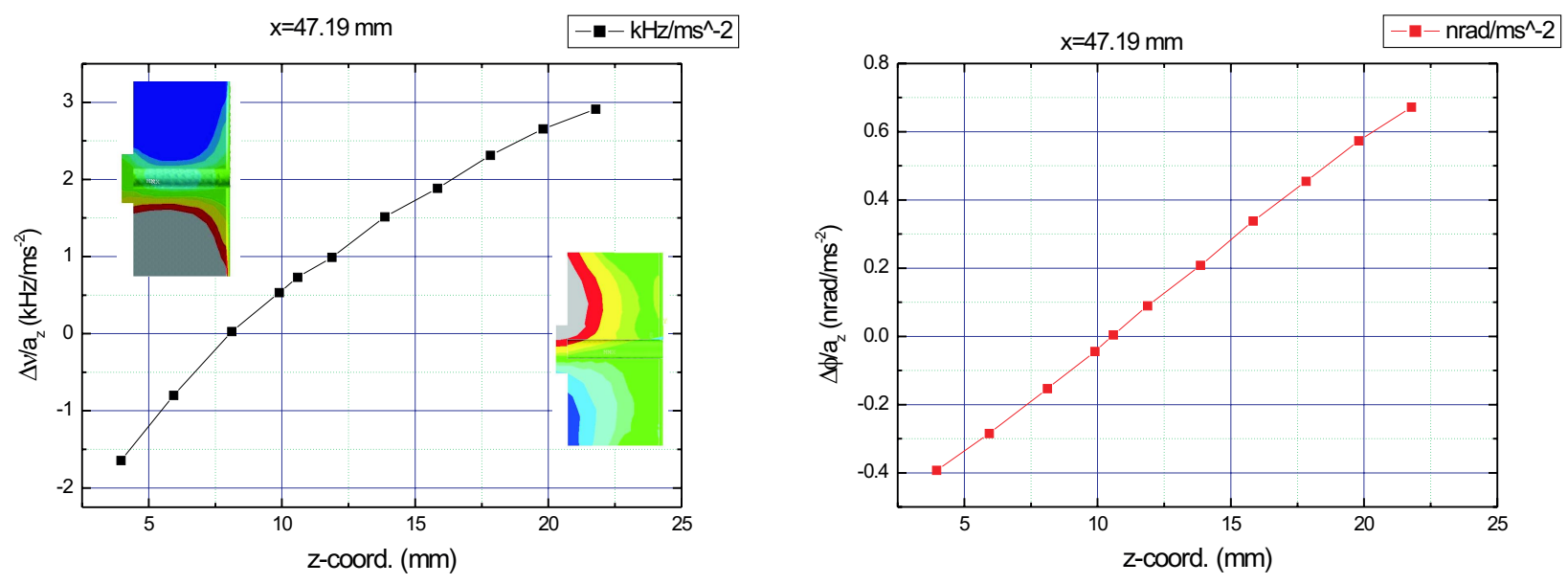

Figure 4. Calculation of frequency displacement of the resonant frequency of the cavity due to vertical acceleration in function of position of the supporting points along the longitudinal (z) axis of the cavity. The origin of the axis is placed on one end of the spacer surface where the mirror is attached. The center of the supporting area is placed at $x=4 \% .19$ mm.

acceleration. To reduce the time of the calculations, only a quarter of the cavity has been calculated, through application of symmetric boundary conditions to the side surfaces.

We found a zero crossing for the displacement sensitivity for a support position of $z_{0}=8.12 \mathrm{~mm}$. In this position, the residual tilt is $\Delta \phi=0.15 \mathrm{nrad} / \mathrm{ms}^{-2}$. Taking into account the slope of the curve in Fig.4, an error $\Delta \mathrm{z}=1 \mathrm{~mm}$ in the position of the support along the z-axis would give a residual vertical sensitivity $1.5 \mathrm{kHz} / \mathrm{ms}^{-2}$. Furthermore for a $\Delta \mathrm{r}=1 \mathrm{~mm}$ displacement error in the axis of the two mirrors (factory specifications) the residual frequency displacement due to tilt effect is $0.6 \mathrm{kHz} / \mathrm{ms}^{-2} \cdot{ }^{15,16}$ The overall frequency shift expected for a cavity held at $z_{0}$ is of the order of $2 \mathrm{kHz} / \mathrm{ms}^{-2}$ for acceleration along the vertical axis. With similar calculations the expected frequency displacement for horizontal acceleration is $10 \mathrm{kHz} / \mathrm{ms}^{-2}$.

For a typical vibration noise spectrum in a lab approaching $1 \mu \mathrm{g} / \sqrt{\mathrm{Hz}}$, this value of frequency sensitivity, sets a limit on frequency noise density of the order of $10 \mathrm{mHz} / \sqrt{\mathrm{Hz}}$, well below the typical thermal noise limit for rigid cavities. ${ }^{17}$

The thermal noise limit (see Fig.7) of our cavities has been estimated by taking noise values for ULE, fused silica and mirror coating as reported in the literature. ${ }^{17}$ This value is about 3 times smaller with respect to the noise level in vertical cavities realized at the same wavelength for similar purposes ${ }^{18}$ which yields a fractional frequency stability of $3.7^{*} 10^{-16}$. 


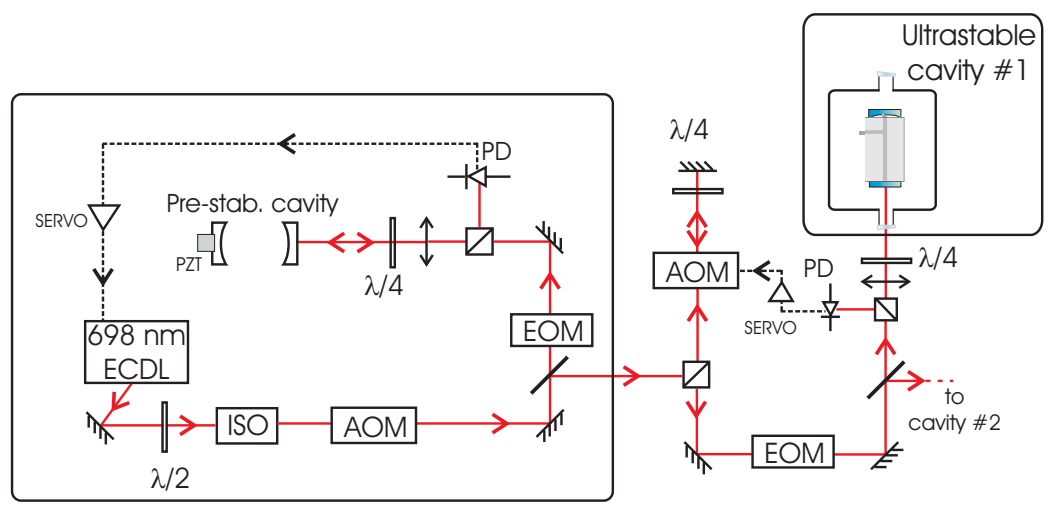

Figure 5. Experimental setup for the stable 698 nm laser, ECDL: extended cavity diode laser, AOM: acusto-optic modulator, ISO: 30 dB optical isolator, EOM: electro-optic modulator.

\section{STABLE LASER AT 698 NM}

The scheme of our stable master laser source for spectroscopy of the intercombination ${ }^{1} \mathrm{~S}_{0^{-}}{ }^{3} \mathrm{P}_{0}$ transition in strontium atoms is reported in Fig. 5. The master laser is an extended cavity $698 \mathrm{~nm}$ diode laser (Littrow configuration). The diode operates around $40{ }^{\circ} \mathrm{C}$ and delivers about $10 \mathrm{~mW}$ of optical power at $82 \mathrm{~mA}$. The reduction of the laser linewidth is performed with a two-step Pound-Drever-Hall frequency stabilization to optical cavities: first, we reduce the linewidth to $<1 \mathrm{kHz}$ by locking the laser to a resonance of a pre-stabilization cavity, and then we further reduce the linewidth by locking the pre-stabilized laser to a resonance of an ultra high finesse cavity. ${ }^{18,19}$

The first pre-stabilization cavity is realized with an invar spacer sitting on a V-shaped aluminium block and has a finesse of $10^{4}$. The resonance is about $150 \mathrm{kHz}$ wide. The servo signal is sent to the PZT control of the laser extended cavity up to $5 \mathrm{kHz}$, and directly to the diode current control with a bandwidth of about $2 \mathrm{MHz} .{ }^{11,20}$ The finesse of the high finesse cavity has been deduced both by measuring the photon cavity lifetime $\tau=43(2) \mu \mathrm{s}$ and by directly observing the linewidth $\Delta \nu=3.7(0.5) \mathrm{kHz}$ of the $\mathrm{TEM}_{00}$ mode of the cavity. Two independent measurement give for both cavities a finesse of $4.1^{*} 10^{5}$ within $4 \%$ of error, corresponding to $7 \mathrm{ppm}$ total losses for each mirror and a cavity quality factor of $1.2^{*} 10^{11} .^{21}$

The second stabilization loop acts at low frequencies (up to $1 \mathrm{kHz}$ ) on the PZT of the pre-stabilization cavity to compensate for low frequency drifts, and at high frequency (up to $50 \mathrm{kHz}$ ) to the AOM used to shift the frequency of the laser through the driving RF frequency generator. The power coupled into the high finesse cavity, when the laser is locked to the lowest $\mathrm{TEM}_{00}$ mode of the cavity is about $60 \%$, while the transmission is typically of the order of $15 \%$. This transmission value is consistent with the measured mirror losses.

In Fig. 7 we show reported the frequency noise of the $698 \mathrm{~nm}$ clock laser source, which was measured by sending part of the light, frequency shifted with an AOM, to the second independent cavity sitting on the same 


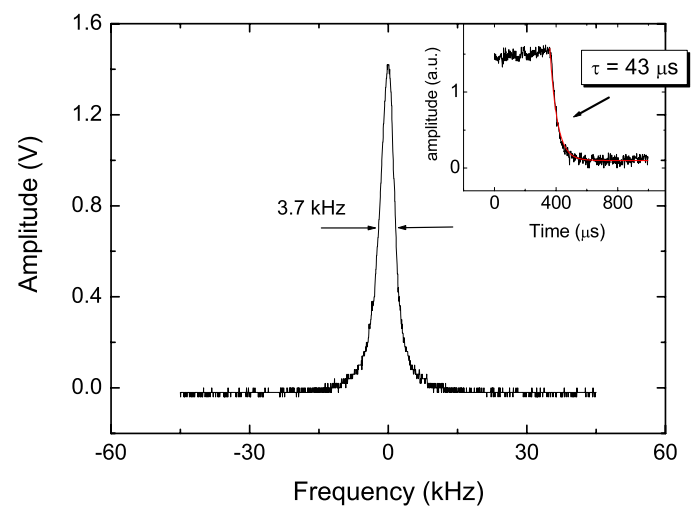

Figure 6. Transmission of the cavity by sweeping across the principal resonant mode of the cavity. In the inset the corresponding ring-down signal is shown. The two measurement gives an estimation for the cavity finesse of $4.1^{*} 10^{5} \pm 10 \%$.

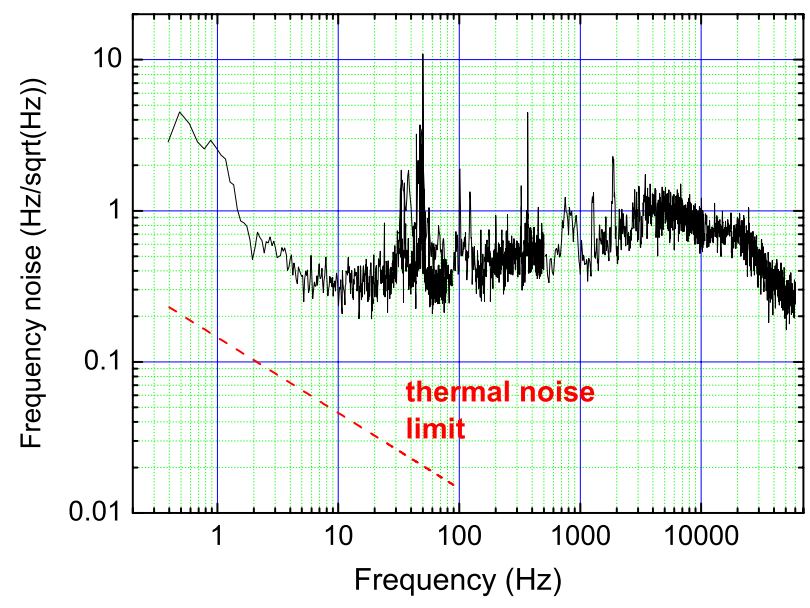

Figure 7. Frequency noise density of the stable $698 \mathrm{~nm}$ laser source locked to the high finesse cavity. The dashed line represents the calculated thermal noise limit due to the contribution of the ULE spacer, the $\mathrm{SiO}_{2}$ mirror substrate and the $\mathrm{Ta}_{2} \mathrm{O}_{5} / \mathrm{SiO}_{2}$ coating .

optical table, and analyzing the error signal obtained when the frequency of the beam is steered around the resonance of the second cavity.

The laser is far from being limited by the thermal noise contribution of the cavity, and optimization of the servo loop parameters and reduction of seismic and acoustic noise contribution is under development. From the analysis of the error signal on the second stabilization loop, we can calculate a laser linewidth of the order of 1 $\mathrm{Hz}$.

To check independently this value and the residual cavity drifts due to temperature variations we also lock 


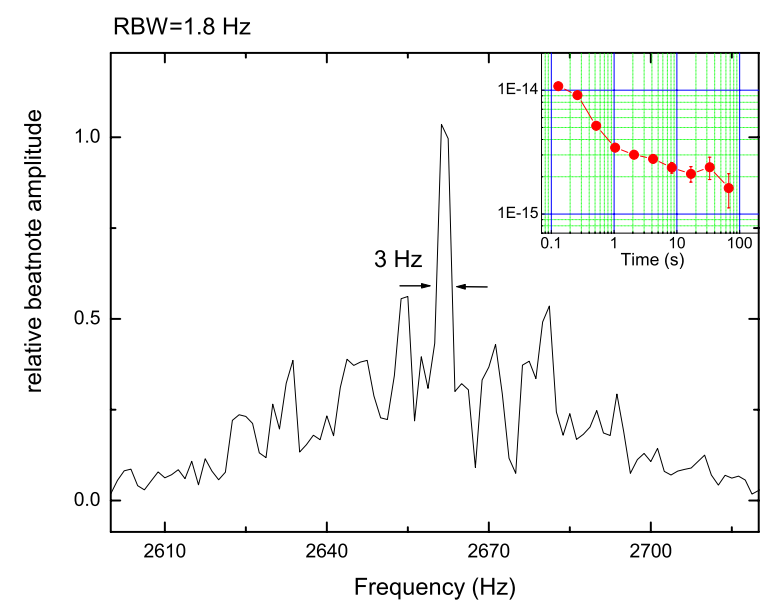

Figure 8. Beatnote of two beams frequency locked independently to two resonances of independent cavities. The linewidth observed with a resolution bandwidth of $1.8 \mathrm{~Hz}$ is about $3 \mathrm{~Hz}$. In the inset is shown the Allan variance of the counted beatnote (0.1 s counter gate time) after removal of residual drift.

the frequency of the light to the second cavity as well generate a beatnote between the two beams which are independently locked to the resonances of the respective cavities. The recorded beat note is reported in Fig. 8 . For convenience the frequency has been down-converted from $200 \mathrm{MHz}$ to about $3 \mathrm{kHz}$. In the inset is shown the Allan variance calculated after removing the linear drift (residual drift of $4 \mathrm{~Hz} / \mathrm{s}$ due to residual thermal control instability) with a computer controlled RF generator.

A preliminary measurement of the thermal expansion coefficient near room temperature has been done by changing the temperature of one of the cavities while maintaining the other one stabilized and measuring the relative shift in the $\mathrm{TEM}_{00}$ mode of the two cavities. We found a mean value of $510^{-8} \mathrm{~K}^{-1}$ for the CTE (Coeff. of Thermal Expansion) in the temperature range of $22-25^{\circ} \mathrm{C}$. This value is larger than what expected from ULE specification, and might be due to the residual temperature expansion coefficient of the two $\mathrm{SiO}_{2}$ mirrors. ${ }^{22}$

Ultimately, we checked the sensitivity to acceleration of the stabilized laser system, by observing the frequency noise imposed into the laser by acceleration in both vertical and horizontal directions. The results of the measurements have been presented in Fig.9. The acceleration noise has been measured with a triaxial (Kinemetrics Episensor), while the frequency noise has been measured by using a resonance of the second high finesse cavity as a frequency discriminator. The frequency noise has been recorded with and without an air damping system applied to the optical table where the laser is mounted. For the frequency noise measurement done without the floating legs, it is possible to recognize two distinct peaks respectively centered around 10 and 30 $\mathrm{Hz}$, corresponding to the two main contributions of acceleration noise in the horizontal and vertical directions. Comparing the peak values at these two frequencies with the vibration noise measured, we found a value of 

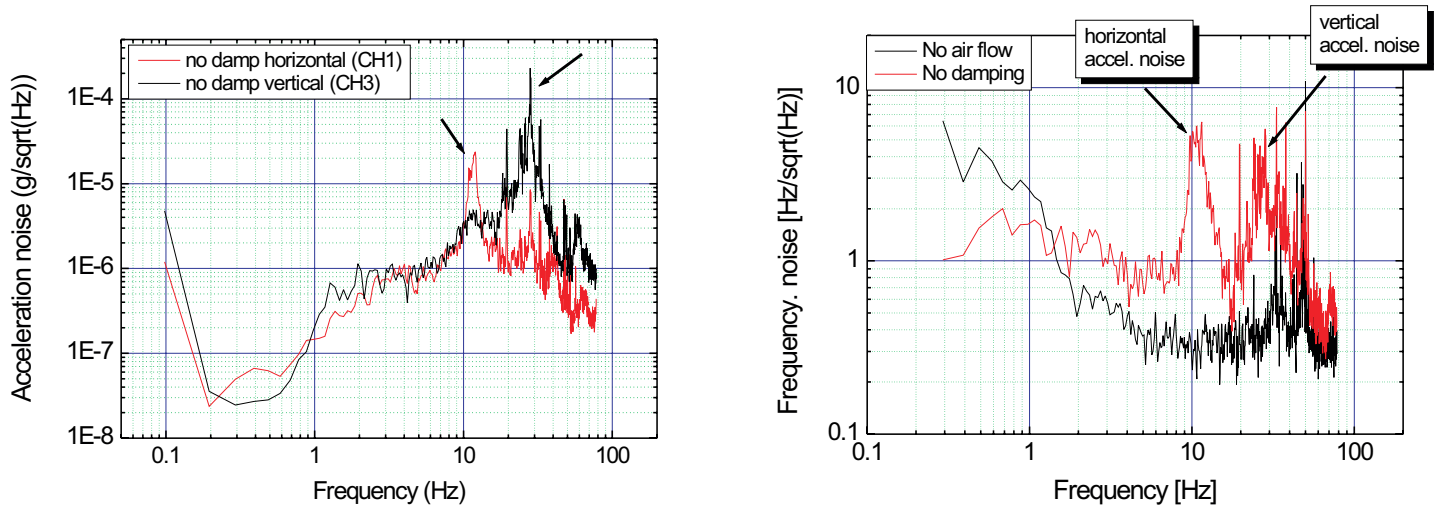

Figure 9. Measurement of the acceleration noise (left) and laser frequency noise density (right) measured with floating optical table and without floating system. From the analysis of the corresponding peaks observed without the floating system, it is possible to estimate the sensitivity to vibration of the clock $698 \mathrm{~nm}$ laser. The two values of $3 \mathrm{kHz} / \mathrm{ms}^{-2}$ and $20 \mathrm{kHz} / \mathrm{ms}^{-2}$ for the sensitivity respectively for vertical and horizontal directions, are in good agreement with the results of our FEM simulations.

$3 \mathrm{kHz} / \mathrm{ms}^{-2}$ and $20 \mathrm{kHz} / \mathrm{ms}^{-2}$ for the sensitivities respectively for vertical and horizontal directions, in good agreement with the results of our FEM simulations.

\section{INFRARED LASER AT 813 NM FOR TRAPPING STRONTIUM AT MAGIC WAVELENGTH}

We developed and tested an all-semiconductor $813 \mathrm{~nm}$ light source to trap atoms in an optical lattice. The source is based on an extended cavity AR coated diode laser delivering up to $40 \mathrm{~mW}$ at $813 \mathrm{~nm}$ and a tapered amplifier. With typical injected optical power of $20 \mathrm{~mW}$ and a driving current of about $1.6 \mathrm{~A}$ it is possible to obtain $600 \mathrm{~mW}$ at the output of the tapered amplifier at $813 \mathrm{~nm}$. About half of that power is then coupled into a polarization maintaining fiber, delivering up to $300 \mathrm{~mW}$ to the atoms in a standing wave configuration. The whole source is mounted on a $50 \mathrm{~cm}$ x $50 \mathrm{~cm}$ breadboard. With this optical configuration we could transfer more than $10 \%$ of the atoms from the MOT operating on the ${ }^{1} \mathrm{~S}_{0^{-}}{ }^{3} \mathrm{P}_{1}$ transition (see Fig. 1). We observed a trapping lifetime of $2 \mathrm{~s}$, limited by background collisions. This source represents an attractive alternative to more expensive and more complex Ti:Sa laser systems used so far to trap strontium neutrals at the magic wavelength. ${ }^{23}$

\section{CONCLUSION}

We presented new laser sources and an experimental apparatus to be used for precision spectroscopy of intercombination lines of strontium. All the laser sources employed to cool, trap and interrogate Sr neutral atoms 


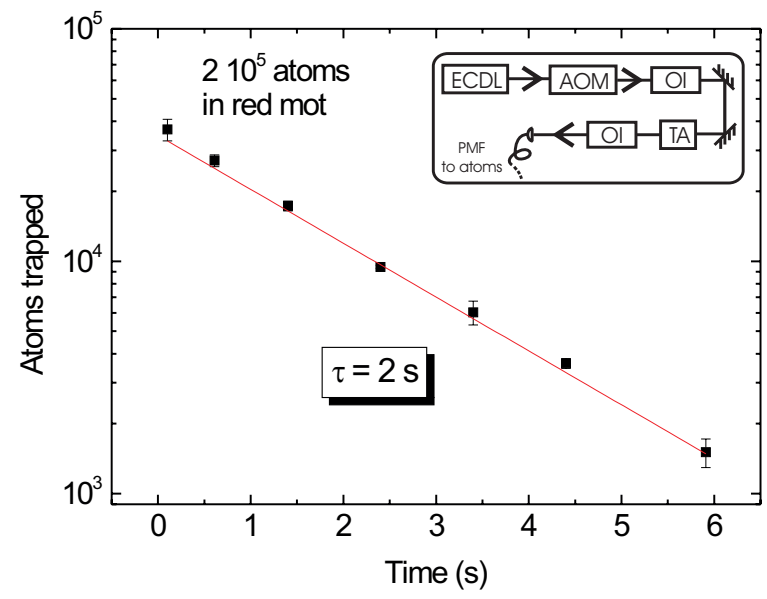

Figure 10. Measurement of the lifetime ${ }^{88} \mathrm{Sr}$ atoms trapped in the $813 \mathrm{~nm} 1 \mathrm{D}$ lattice. In the inset the laser setup is presented. ECDL, Extended cavity diode laser, AOM, acusto-optical isolator, OI optical isolator, TA tapered amplifier.

use only semiconductor devices. The compactness and reliability of such sources represent one of the first steps towards the realization of a transportable optical frequency reference to be employed in fundamental and applied physics on Earth and in Space.

We acknowledge the contribution of B. Pinto Da Sousa in the early stage of the experiment. We thank C. Oates for his careful reading of the manuscript. This work is supported by ESA under contract 19838/06/F/VS (2006), ASI under contract 1/013/06/0 (2006) and LENS.

\section{REFERENCES}

1. C. Oates, Proc. of the Joint 2007 EFTF \& IEEE-FCS (TimeNav'07), (2007)

2. J. Ye, Proc. of the Joint 2007 EFTF \& IEEE-FCS (TimeNav'07), (2007)

3. J. Bergquist, Proc. of the Joint 2007 EFTF \& IEEE-FCS (TimeNav'07), (2007)

4. see for example the EGE and SAGAS proposal in the framework of ESA Cosmic Vision (2007)

5. Dusseldorf University, LENS-Universita di Firenze, SYRTE Obs. De Paris, PTB Space Optical Clocks ESA CONTRACT (2007)

6. S. Schiller et al. "Precision test of General Relativity and the Equivalence Principle using ultrastable optical clocks: a mission proposal" Proc. of the 39th ESLAB Symposium "Trends in Space Science and Cosmic Vision 2020", pp.39-42, F. Favata, J. Sanz-Forcada, A. Gimenez, eds., (ESA SP-588, 2005)

7. S. Schiller et al. "Optical clocks in space" Proceedings III International Conference on Particle and Fundamental Physics in Space (SpacePart06), Beijing 19-21 April (2006). 
8. Kayser Italia S.r.l, LENS- Università di Firenze, Dusseldorf University, SYRTE Obs. De Paris, OAC-Study on the feasibility and applications of optical clocks as frequency and time references in ESA Deep Space Stations, ESA CONTRACT 19838/06/F/VS (2006)

9. N. Poli, R. E. Drullinger, G. Ferrari, J. Léonard, F. Sorrentino, and G. M. Tino, "Cooling and trapping of ultracold strontium mixtures", Phys. Rev. A 71, 061403(R) (2005)

10. F. Sorrentino, G. Ferrari, N. Poli, R. Drullinger, G. M. Tino, "Laser cooling and trapping of atomic strontium for ultracold atoms physics, high-precision spectroscopy and quantum sensors", Mod. Phys. Lett. B 20, 1287 (2006)

11. N. Poli, G. Ferrari, M. Prevedelli, F. Sorrentino, R. E. Drullinger, G. M. Tino, "Laser sources for precision spectroscopy on atomic strontium", Spectrochim. Acta Part A 63, 981 (2006)

12. Kayser Italia S.r.l., LENS- Universita' di Firenze, "ORA- Studio di fattibilita' per orologi atomici ottici", ASI CONTRACT 1/013/06/0 (2006),

13. T. Rosenband private comm. (2006)

14. L. Chen, J. L. Hall, J. Ye, T. Yang, E. Zang, and T. Li "Vibration-induced elastic deformation of Fabry-Perot cavities", Phys. Rev. A 74, 053801 (2006)

15. T. Nazarova, F. Riehle, U. Sterr, "Vibration-insensitive reference cavity for an ultra-narrow-linewidth laser", Appl. Phys. B 83, 531 (2006)

16. A.E. Siegman, Lasers (University Science Books, Mill Valley, CA, 1986)

17. K. Numata, A. Kemery, and J. Camp, "Thermal-Noise Limit in the Frequency Stabilization of Lasers with Rigid Cavities", Phys. Rev. Lett. 93, 250602 (2004)

18. A. D. Ludlow, X. Huang, M. Notcutt, T. Zanon-Willette, S. M. Foreman, M. M. Boyd, S. Blatt, J. Ye, "Compact, thermal-noise-limited optical cavity for diode laser stabilization at 110-15", Opt. Lett. 32, 641 (2007)

19. B. C. Young, F. C. Cruz, W. M. Itano, and J. C. Bergquist, "Visible Lasers with Subhertz Linewidths", Phys. Rev. Lett. 82, 3799 (1999)

20. G. Ferrari, P. Cancio, R. Drullinger, G. Giusfredi, N. Poli, M. Prevedelli, C. Toninelli, and G. M. Tino, "Precision Frequency Measurement of Visible Intercombination Lines of Strontium", Phys. Rev. Lett. 91, $243002(2003)$

21. G. Rempe, R. J. Thompson, H. J. Kimble, R. Lalezari, "Measurement of ultralow losses in an optical interferometer", Opt. Lett. 17, 363 (1992)

22. G. Santarelli, priv. comm.

23. M. Takamoto, F.-L. Hong, R. Higashi and H. Katori, "An optical lattice clock", Nature 435, 321 (2005) 